\title{
Ballooning Your Way in Altered Anatomy ERCP: Ready for Deductive Learning
}

\author{
Smit S. Deliwala ${ }^{1} \cdot$ Saurabh Chawla ${ }^{2}$ \\ Accepted: 23 November 2021 / Published online: 10 January 2022 \\ (C) The Author(s), under exclusive licence to Springer Science+Business Media, LLC, part of Springer Nature 2021
}

In 1987, Lewis et al. used a balloon to 'inchworm' through the small bowel during a time when intraoperative endoscopy and guide-string were the only reliable methods, thus revolutionizing total small bowel visualization and access [1]. Over the next decade, refinements in technology to facilitate endoscopic small bowel access led to the introduction of several deep enteroscopy devices, of which single and deep balloon enteroscopes are the most widely used [2]. Double balloon enteroscopy (DBE) utilizes a specially designed long endoscope $(230 \mathrm{~cm})$ with a balloon attachment at the tip, which can be advanced through a long overtube with another balloon on it. After the initial deep advancement with the enteroscope, the distal tip is anchored in place with pressure-monitored balloon inflation, and the overtube is advanced to the tip of the endoscope. The second overtube balloon is then inflated, and the apparatus is gently withdrawn to pleat the small bowel and straighten the loops. Subsequent advancement is done by sequentially deflating balloons, advancing the enteroscope and overtube with repeated reductions, commonly referred to as the "push and pull" technique. Single balloon enteroscopy (SBE) is a modification of the double balloon enteroscopy wherein only the overtube has the balloon and was introduced to streamline this multi-step procedure.

Although deep enteroscopy was proposed initially for luminal diagnostic and therapeutic indications in the middistal small bowel, in 2005, Haruta et al. published the first successful double balloon-assisted choledochal jejunal ERCP with anastomotic stricture dilation in a 7-year-old boy who had undergone a Roux-en-Y living donor liver

Saurabh Chawla

saurabh.chawla@emory.edu

1 Department of Medicine, Michigan State University at Hurley Medical Center, Flint, MI, USA

2 Division of Digestive Diseases, Department of Medicine, Emory University School of Medicine, Atlanta, GA 30322, USA transplant 6 years prior [3]. This was a significant advance since until then, patients with surgically-altered anatomy would require staged percutaneous or complicated surgical procedures for the management of pancreaticobiliary disorders, which otherwise could be managed endoscopically in patients with conventional anatomy.

Since 2005, there has been ongoing interest and innovation within deep enteroscopy-assisted pancreaticobiliary access in patients with altered gastrointestinal anatomy. Balloon-assisted ERCP (BAE-ERCP), as this technique is commonly termed, is a unique solution for patients with altered anatomy such as Roux-en-Y reconstructions (RY), hepaticojejunostomy (HJ), Billroth II gastrectomy (B-II), and pancreaticoduodenectomy (PD) that require pancreaticobiliary access and therapy [4]. As elegant as this appears, there are several practical challenges when performing these procedures. These include varying lengths of the pancreaticobiliary limb, sharp angulations in the bowel due to adhesions or internal herniations, and irreducible fixed loops that can preclude access to the region of interest. In patients with native ampullas, the orientation of the biliary and pancreatic axes is reversed, complicating cannulation of the ducts. Furthermore, the balloon enteroscopes are longer with a narrower accessory channel (usually $2.8 \mathrm{~mm}$ ) that does not allow for conventional ERCP catheters and related devices used for these procedures. For these reasons and the relative infrequency of these procedures compared with conventional ERCPs, these procedures are not widely performed, being relegated to specialized centers where a few endoscopists have developed expertise in these techniques. Consequently, training in BAE-ERCPs is not standardized, with no validated means of determining competency.

In this issue of Digestive Diseases and Sciences, Hosono et al. report a very interesting retrospective analysis of 687 SBE-ERCPs performed by seven expert endoscopists who had performed $>400$ ERCPs each with no significant prior experience in balloon enteroscopy [5]. In their cohort, the most common reconstruction technique was a Roux-en-Y 
hepaticojejunostomy (HJ) in $49 \%$ of patients, followed by pancreaticoduodenectomy (PD) in 23\%, Roux-en-Y gastrectomy (R-Y) in $18 \%$, and Billroth II gastrectomy (B-II) in $8 \%$. The authors report an endoscope insertion success rate (which they define as successfully reaching the target) in $95 \%$ of patients (650/687) and an overall success rate of $92 \%$ (634/687). In their institution, if an endoscopist could not reach the area of interest within $30 \mathrm{~min}$, another endoscopist would endeavor to complete the procedure. Using this metric, the procedural success rate per endoscopist was reported as $83.5 \%$. The overall adverse event rate was $6.3 \%$, with pancreatitis $(1.7 \%)$ and perforations (1.4\%) the most common complications. Most of the patients with pancreatitis were in the native papilla group (R-Y, B-II), which had a $6 \%$ rate of pancreatitis. The authors did not note any significant difference in insertion or procedural success between different reconstruction types according to subgroup analysis. Importantly, they do not report any difference in cannulation or procedural success rates when comparing native papilla (R-Y, B-II) to bilioenteric anastomosis (PD and HJ), although this may be under-powered since only $174 / 670$ patients had native ampullas. The procedures were done efficiently, with a mean procedural time of $57 \mathrm{~min}$, whereas procedures in the R-Y group took longer (72 $\mathrm{min}$ ), presumably due to the longer length of the Roux limb.

The authors then used this exhaustive dataset to evaluate the learning curves for achieving competence in SBEERCPs among their group of endoscopists by systematically studying outcomes in blocks of ten procedures (based on the Osborn-Parnes creative process amongst trainees) [6]. They defined competence as $>90 \%$ success with endoscope insertion and with procedure completion, each analyzed separately. According to logistic regression, the success rates for insertion and completion significantly increased over time with experience, with 20 cases required to achieve $>90 \%$ insertion success and 30 cases required to achieve $>90 \%$ procedural success. Based on this analysis, the authors propose a target of 30 SBE-ERCP procedures in order to achieve competence in this technically challenging procedure.

Previously, there have been several prospective and retrospective studies and two well-designed meta-analyses evaluating outcomes of SBE-ERCP. In 2015, Inamdar et al. published a meta-analysis of 15 studies conducted between 2009 and 2014 which included 461 patients, reporting an enteroscopy success rate of $81 \%$ (55-100\%), a procedural success rate of $62 \%(60-78 \%)$, and an adverse event rate of $6.5 \%(4.7-9 \%)$ [7]. One recent advance in SBE-ERCPs is the development of a shorter length SBE (model SIF-Y0004; Olympus Medical Systems, Tokyo, Japan, working length$152-\mathrm{cm})$ with a large accessory channel $(3.2 \mathrm{~cm})$ facilitating deep insertion while enabling the use of conventional ERCP accessory devices. An updated systematic review and meta-analysis by Tanisaka et al. in 2021 included 21 studies, analyzing a pool of 1227 SBE-ERCPs. They reported mildly improved outcomes (as compared with the prior meta-analysis) with an enteroscopy success of 86.6\% (52.6-100\%), procedural success $75.8 \%$ (71-80.3\%), and a similar adverse event rate $6.6 \%(5.2-8.2 \%)$ [8]. Notably, in this meta-analysis, six studies used the short SBE-ERCP which was associated with superior procedural success rates ( $82 \%$ vs. $73 \%$ according to subgroup analysis).

In light of the prior studies, this study by Hosono et al. is remarkable in several ways. Although retrospective, it is well-designed, demonstrating the most extensive singlecenter experience published to date. Despite using the long length SBE $(200 \mathrm{~cm})$ and performing these procedures under conscious sedation, the outcomes reported in this study are far superior to those reported in most prior publications. These improved outcomes may be directly ascribed to the elegantly done learning curve analysis, demonstrating improved outcomes with increased experience. This finding is externally validated by the results of prior publications in which case series with fewer patients report lower procedural success rates [9]. Furthermore, there is internal validity since the learning curves were similar for all seven included endoscopists.

Prior to generalizing the authors' experience, several points should be emphasized: (1) Most patients in this series underwent the procedures for biliary and not pancreatic indications; (2) A significant proportion of patients in this cohort (PD, B-II, H-J) have relatively shorter biliary-pancreatic limbs where endoscopic insertion can frequently be established with shorter forward-viewing endoscopes or duodenoscopes unless limited by sharp angulation [10]; (3) None of the included patients had Roux-en-Y gastric bypass, which is probably one of the most frequently encountered altered anatomy ERCPs currently encountered in the Western hemisphere that are frequently associated with a lower success rate of BAE-ERCP [4]. Likewise, therapeutic interventions such as stent placement, stone extraction, and drainage were not well described despite assessing procedure success. As mentioned before, these therapeutic maneuvers require special long-length devices, and therefore, it is imperative to ensure that the endoscopy unit has these in stock prior to embarking on these procedures. Another key point to remember is that the endoscopists in this study were experts in ERCP techniques and may have needed fewer procedures in in order to master SBE-ERCP. Therefore, it may be reasonable to presume that the learning curve may be longer in trainees, requiring further studies to support this contention.

In summary, altered anatomy ERCPs are technically challenging and are associated with variable outcomes. Unfortunately, if unsuccessful, the alternatives for patients are procedures with higher risk and morbiditity. Therefore, this study by Hosono et al. is a landmark study in the field of BAE-ERCPs as it establishes a systematic approach to 
achieving competence in SBE-ERCPs, demonstrating that this approach is associated with outcomes on par with conventional ERCPs.

Funding No financial disclosure to declare.

\section{Declarations}

Conflict of interest The authors have no conflict of interest to declare.

\section{References}

1. Lewis BS, Waye JD. Total small bowel enteroscopy. Gastrointest Endosc 1987;33:435-438.

2. ASGE Standards of Practice Committee, Khashab MA, Pasha SF, et al. The role of deep enteroscopy in the management of smallbowel disorders. Gastrointest Endosc. 2015;82:600-607.

3. Haruta H, Yamamoto H, Mizuta K et al. A case of successful enteroscopic balloon dilation for late anastomotic stricture of choledochojejunostomy after living donor liver transplantation. Liver Transpl 2005;11:1608-1610.

4. Barakat MT, Adler DG. Endoscopy in patients with surgically altered anatomy. Am J Gastroenterol 2021;116:657-665.
5. Hosono K, Sato T, Hasegawa S, et al. Learning curve of endoscopic retrograde cholangiopancreatography using single balloon enteroscopy. Dig Dis Sci. (Epub ahead of print). https://doi.org/ 10.1007/s10620-021-07342-2.

6. Ekkelenkamp VE, Koch AD, Rauws EAJ et al. Competence development in ERCP: the learning curve of novice trainees. Endoscopy 2014;46:949-955.

7. Inamdar S, Slattery E, Sejpal DV et al. Systematic review and meta-analysis of single-balloon enteroscopy-assisted ERCP in patients with surgically altered GI anatomy. Gastrointest Endosc 2015;82:9-19.

8. Tanisaka Y, Ryozawa S, Mizuide M et al. Status of single-balloon enteroscopy-assisted endoscopic retrograde cholangiopancreatography in patients with surgically altered anatomy: systematic review and meta-analysis on biliary interventions. Dig Endosc 2021;33:1034-1044.

9. Moreels TG. Altered anatomy: enteroscopy and ERCP procedure. Best Pract Res Clin Gastroenterol 2012;26:347-357.

10. ASGE Standards of Practice Committee, Faulx AL, Lightdale JR, et al. Guidelines for privileging, credentialing, and proctoring to perform GI endoscopy. Gastrointest Endosc. 2017;85:273-281. (published correction appears in Gastrointest Endosc. 2017 May;85(5):1115).

Publisher's Note Springer Nature remains neutral with regard to jurisdictional claims in published maps and institutional affiliations. 